transplantation [LT]/death, and all-cause mortality) were stratified according to age strata at UC diagnosis.

Results Over 10 years, 128694 incident UC cases were identified (annualised incidence/100,000 population: 23.8 in 2006; rising to 25.1 in 2015). Of this group, 2124 were diagnosed with PSC at some point (incidence in 2006 and 2015: 0.29 and 0.4 , respectively). Observing the UC cohort in entirety, we observed 2101 st LT (206 in group 2), 9413 individuals who came to colectomy, 1,208 CRC cases, and 11177 pt. deaths. The leading cause of mortality was coronary disease (1\%) in group 1; whereas liver-related death (5.9\%), cholangiocarcinoma $(4.6 \%)$ and CRC $(1 \%)$ predominated in group 2. The incidence rate ([IR]/1000-pt.yrs.) was greater in the UC/PSC group for colectomy (17.3 vs 13.7), CRC (5.6 vs 1.5 ), LT/death (38.5 vs 15.1 ), and all cause mortality (26.4 vs 15.1); $\mathrm{p}<0.001$ for all. Time-dependent Cox regression validated the negative impact of PSC onset for each endpoint (time-dependent adjusted hazard ratio: 1.62, 3.31, 2.47 and 1.62, respectively; $\mathrm{p}<0.001$ for all). Compared to UC alone, the standardised incidence ratio (SIR) for CRC was greatest in UC/PSC of young presenting age $(<40 \mathrm{y}$ ) ; a 7 -fold increase (figure 1A). This contrasted to pts. diagnosed above age 40 (SIR 4). Although absolute mortality rate was elevated in older ages (figure 1B) it was in young pts. with UC/PSC that the contrast vs UC alone was most evident for 5 year. $(1.6 \%$ vs $0.4 \%)$ and 10 year. survival $(3.6 \%$ vs $0.6 \%)$; a 4 and 6 fold increase, respectively. Indeed, standardised mortality (SMR) was the greatest for patients diagnosed age $\leq 40$ years., and plateaued with older age at diagnosis (figure 1C).
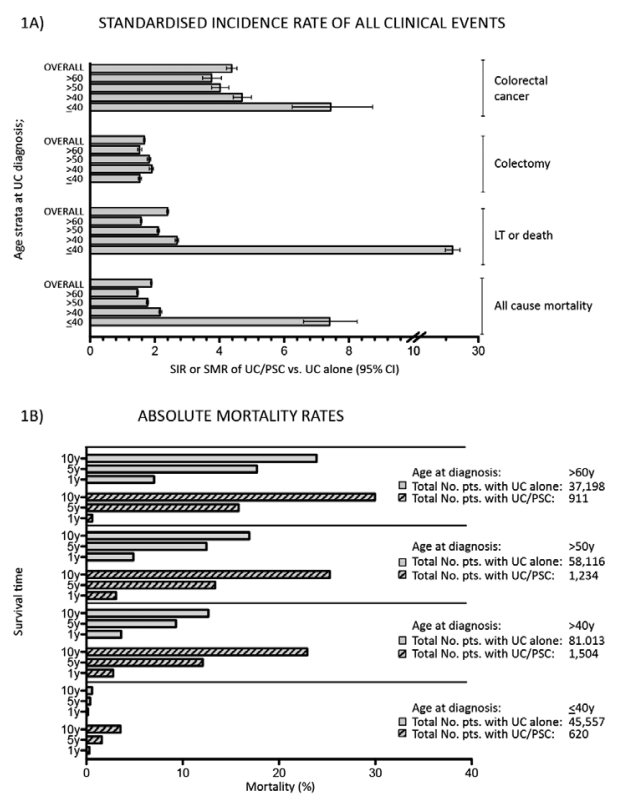

1C) STANDARDISED MORTALITY RATE OVER TIME PER AGE GROUP

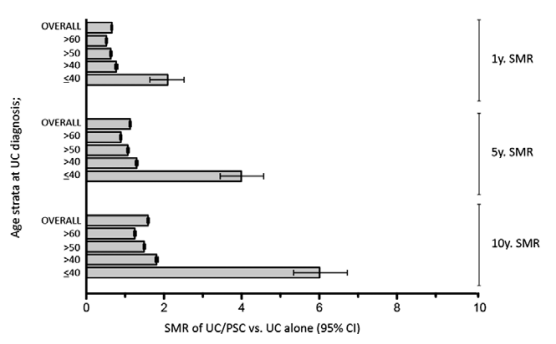

Abstract OWE-012 Figure 1 a) standardised incidence rate of all clinical events b) absolute mortality rates c) standardised mortatility rate over time per age group
Conclusion In pts. diagnosed aged $\leq 40$ years. with UC, development of PSC is associated with 6-fold increase in mortality and 7-fold increased risk of CRC. Within IBD cohorts, those diagnosed at a young age with PSC have a heightened and disproportionate unmet need for life-prolonging therapies.

\section{OWE-013 DEVELOPING AN AUTOMATED INTELLIGENT LFT (ILFT) DIAGNOSTIC ALGORITHM - IMPROVED OUTPUT FOR LESS TRIBULATION}

\footnotetext{
${ }^{1,2}$ Paul Brennan*, ${ }^{1,2}$ Emma Robinson, ${ }^{2}$ Michael Miller, ${ }^{3}$ Kathleen Boyd, ${ }^{2}$ Ellie Dow ${ }^{1,2}$ John Dillon. ${ }^{1}$ University Of Dundee, Dundee, UK; ${ }^{2}$ NHS Tayside, Dundee, UK; ${ }^{3}$ University of Glasgow, Glasgow, UK
}

\subsection{6/gutjnl-2018-BSGAbstracts.206}

Introduction Liver Function Tests (LFTs) are commonly abnormal, however; the diagnostic approach to individuals with deranged LFTs is variable, with lengthy processes and an increasing number of referrals to tertiary services and sub-optimal investigation of many patients. The aim of the project was to improve diagnostic proficiency, improving quality of investigation, reducing overall costs to practitioners and patients and reducing secondary care referrals.

Methods The project developed a functional automated intelligent LFT' (iLFT) system.

This algorithm uses the combination of diagnostic criteria for liver disease, an investigation ordering and reporting system, and the tracked blood sciences system. iLFT produces a diagnosis or description of the abnormality with staging information and suggestions for further management. In general allowing allocation to 3 broad outcomes series of outcomes; a) diagnosis requiring complex treatment or advanced liver disease, b) a diagnosis of early or simple liver disease, c) where a clear diagnosis is not made; the GP receives staging and prognostic information including referral criterion.

A step wedge design trial was conducted in 6 GP practices (covering 30000 patients). Patients with LFTs measured in the previous 6 months with abnormalities were retrospectively used as controls. During the intervention period (6 months); GPs requested the iLFT option and those patients with abnormal LFTs were assessed.

Results Of 719 patients recruited, (Controls=490; interventional group $=229$ ) the iLFT system increased the diagnosis liver disease from first test abnormal LFT cohorts from 16\% to $56 \%$. The adjusted (for the step wedge design) difference in rate of liver disease diagnosis was a highly significant increase of $43 \%$ (95\% CI 27\%, 59\%)

Health economic analysis showed an incremental careequivalent ratio (ICER) of $£ 284$ and over a patient lifetime increased quality adjusted life years and saving the NHS (or equivalent healthcare providers) an average $£ 3216$ per patient - an unequivocally dominant strategy.

Conclusions iLFT increases liver disease diagnosis, improving quality of care and is unequivocally cost effective. These outcomes can be achieved with minor changes to working practices and existing lab infrastructure and ultimately aim to Result in appropriation of individuals being managed in the most apposite clinical infrastructure. 